\title{
Efeito da Concentração na Ascensão de Soluções Preservativas Preparadas com CCB em Moirões de Eucalyptus
}

\author{
Juarez Benigno Paes ${ }^{1}$, Dercílio Junior Verly Lopes ${ }^{2}$, Fabricio Gomes Gonçalves ${ }^{1}$, \\ Flávia Maria Silva Brito ${ }^{2}$, Lucas Recla Lombardi ${ }^{3}$ \\ ${ }^{1}$ Departamento de Ciências Florestais e da Madeira, Universidade Federal do Espírito Santo - UFES, \\ Jerônimo Monteiro/ES, Brasil \\ ${ }^{2}$ Programa de Pós-graduação em Ciências Florestais, Universidade Federal do Espírito Santo - UFES, \\ Jerônimo Monteiro/ES, Brasil \\ ${ }^{3}$ Programa de Pós-graduação em Ciência Florestal, Universidade Federal de Viçosa - UFV, Viçosa/MG, Brasil
}

\section{RESUMO}

Este trabalho teve o objetivo de verificar o efeito da concentração na ascensão de soluções preservativas no tempo de tratamento em moirões de Eucalyptus urophylla $\times$ Eucalyptus grandis. Foram preparadas soluções com quatro concentrações de ingredientes ativos de borato de cobre cromatado - CCB, com 0,$5 ; 1,0 ; 2,0 ; 3,0 \%$. Foi empregado o método de substituição de seiva por transpiração radial e tratados cinco moirões para cada concentração. O tempo de ascensão para as respectivas concentrações foram de 8, 7, 5 e 3 dias. A penetração foi analisada em quatro posições nos moirões e a retenção, na posição correspondente à zona de afloramento em moirões instalados. As penetrações de boro e cobre foram satisfatórias para os moirões tratados com solução de 3,0\%, tendo aqueles tratados com 2,0\% atingido valores próximos aos recomendados pela legislação brasileira. As retenções nas amostras externas dos discos atingiram valores recomendados, exceto nos moirões tratados com $0,5 \%$. O incremento da concentração da solução causou a diminuição do tempo de tratamento dos moirões.

Palavras-chave: madeira tratada, moirão de cerca, substituição de seiva.

\section{Effect of Concentration on Ascension of Preservative Solutions Prepared with CCB in Eucalyptus Fence Posts}

\begin{abstract}
The aim of this study was to verify the influence of concentration on ascension of preservative solutions in the treatment time of Eucalyptus urophylla $\mathrm{x}$ Eucalyptus grandis fence posts. The solutions were prepared with four concentrations of chromated copper borate - CCB, with 0.5; 1.0; 2.0 ; and 3.0\% active ingredients. The sap displacement method by radial transpiration was employed, and five fence posts were treated for each concentration. The ascension times of the respective concentrations were of $8,7,5$, and 3 days. Penetration was analyzed at four positions on fence posts and retention was analyzed at the position corresponding to the ground zone of installed fence posts. Boron and copper penetrations were satisfactory for the fence posts treated with $3.0 \%$ solution, and fence posts treated with $2.0 \%$ solution reached values close to those recommended by the Brazilian legislation. Retention in the samples of external disks reached the recommended values, except for the fence posts treated with $0.5 \%$ solution. Increased solution concentration caused a reduction in the treatment time of fence posts.
\end{abstract}

Keywords: treated wood, fence posts, sap displacement. 
$179-8087.036913$

0980 (impresso) $)^{1 . ~ I N T R O D U C ̧ A ̃ O ~}$

79-8087 (online)

Com o passar do tempo, a legislação brasileira sobre os recursos naturais, principalmente os florestais, tornou-se cada vez mais rigorosa; assim, a exploração das florestas nativas tem uma fiscalização cada vez mais rígida. Deste modo, com o intuito de diminuir a pressão sobre tais florestas, fez-se com que os reflorestamentos tivessem, nas últimas décadas, um crescimento no setor agrário nacional. No entanto, a maioria das espécies de Eucalyptus empregada nos reflorestamentos tem baixa resistência natural a organismos xilófagos (Lelles \& Rezende, 1986; Paes \& Vital, 2000; Paes et al., 2005), havendo a necessidade da aplicação de tratamentos químicos para melhoria da vida útil em serviço das mesmas.

As técnicas de tratamento químico da madeira consistem em introduzir, por meio de processos adequados, produtos químicos na estrutura da madeira, visando torná-la tóxica aos organismos que a utilizam como fonte de alimentos (Brazolin, 2007), a fim de aumentar sua durabilidade em serviço.

A qualidade das madeiras tratadas está diretamente relacionada à retenção, penetração $\mathrm{e}$ distribuição, fatores estes que dependem basicamente da espécie de madeira, do produto preservativo e do processo de tratamento utilizado (Brazolin, 2007). A Norma Brasileira Regulamentadora - NBR 9480 da Associação Brasileira de Normas Técnicas (ABNT, 2009) estabelece que, em função da exposição à qual os moirões de eucalipto tratados com preservantes hidrossolúveis estão dispostos, a retenção mínima de ingredientes ativos deve ser de $6,5 \mathrm{~kg} \cdot \mathrm{m}^{-3}$ e que a penetração ocorra em todo o alburno e na porção penetrável do cerne.

Para o tratamento da madeira, existem os métodos industriais e os não industriais. Dentre os não industriais, o de substituição da seiva é de fácil operacionalidade e baixo custo (Farias Sobrinho et al., 2005). No tratamento, emprega-se madeira verde, roliça e descascada, a qual é disposta verticalmente em tambores, tendo a base submersa na solução. Após o tratamento, os moirões devem passar pelo processo de secagem ao ar, em local coberto e ventilado. Um dos produtos mais utilizados para este fim é o borato de cobre cromatado - CCB (Magalhães \& Pereira, 2003).

Várias pesquisas vêm sendo desenvolvidas com o emprego do método de substituição de seiva; contudo, poucas investigações têm sido realizadas para verificar o efeito da concentração da solução no tempo de ascensão do produto preservativo ao longo da peça. Portanto, uma análise da influência da concentração do produto preservativo no tempo de tratamento de madeiras de Eucalyptus spp., para esse método, é de fundamental importância, proporcionando um padrão na utilização do mesmo, de acordo com a concentração da solução de tratamento utilizada.

Assim, esta pesquisa teve o objetivo de verificar o efeito da concentração da solução preservativa preparada com cobre, cromo e boro (CCB) no tempo de tratamento de moirões de Eucalyptus urophylla $\times$ Eucalyptus grandis.

\section{MATERIAL E MÉTODOS}

\subsection{Obtenção e preparo do material}

As árvores de Eucalyptus urophylla $\times$ Eucalyptus grandis foram colhidas na propriedade rural Providência, situada na Zona Rural de Jerônimo Monteiro, sul do Estado do Espírito Santo, de coordenadas geográficas $20^{\circ} 47^{\prime} 21^{\prime \prime} \mathrm{S}$ e $41^{\circ} 23^{\prime}$ 42 " W, a 780 metros de altitude. O talhão possui aproximadamente 4,5 hectares, localizado em relevo acidentado e espaçamento de plantio variável. As árvores eram jovens, com cerca de sete anos de idade.

Foram abatidas três árvores, com 18 metros de altura em média, sendo estas sadias e com fuste sem defeitos (tortuosidade e bifurcações), com diâmetro entre 12 e $14 \mathrm{~cm}$. Os troncos foram seccionados em peças de 2,20 m de comprimento, obtendo sete a oito peças por árvore.

Após o abate, as peças foram transportadas para o local de tratamento, situado no Departamento de Ciências Florestais e da Madeira da Universidade Federal do Espírito Santo (UFES), descascadas e identificadas de acordo com o tratamento. O tempo entre o abate e o tratamento foi inferior a 24 horas. Cada tratamento foi composto de cinco moirões para cada concentração empregada, com 0,$5 ; 1,0 ; 2,0$; 
$3,0 \%$ de ingredientes ativos (i.a.). Os moirões foram agrupados de modo que os tratamentos tivessem aproximadamente o mesmo volume de madeira, formado por peças provenientes da base, do meio e do topo das árvores.

Para o preparo das soluções preservativas, foi utilizada uma solução composta por sulfato de cobre penta-hidratado, dicromato de potássio e ácido bórico (CCB). As soluções foram preparadas em conformidade com a NBR 9480, ABNT (2009). As quantidades de cada produto químico empregado, para 100 litros de solução, constam da Tabela 1.

Após o preparo, as soluções estoques foram armazenadas em tambores de 200 litros. Antes das reposições das soluções nos tambores, nos quais se encontravam os moirões em tratamento, em função da absorção da solução pela madeira, a solução estoque era homogeneizada.

Os moirões foram dispostos nos tambores e ficaram parcialmente submersos ( $50 \mathrm{~cm}$ da base) na solução preservativa e suas porções áreas mantidas afastadas, a fim de proporcionar boa aeração entre as peças. O tratamento foi realizado em local coberto e ventilado.

Para se evitar a evaporação das soluções preservativas, derramou-se $350 \mathrm{~mL}$ de óleo vegetal em cada tambor, de modo a formar-se uma camada fina sobre as soluções. Diariamente, a quantidade de solução absorvida pela madeira era reposta, mantendo-se constante o nível inicial nos recipientes. Os moirões foram retirados dos tratamentos quando o preservativo atingiu o topo dos mesmos.

$\mathrm{Na}$ medida em que as condições climáticas influenciam no processo de substituição de seiva, durante o tratamento da madeira foi efetuado o monitoramento da temperatura e da umidade relativa locais, uma vez que o tempo de tratamento é menor em dias quentes e secos, e maior em dias frios e úmidos. Neste trabalho, foi efetuada a mensuração do tempo em dias de ascensão do produto preservante CCB até o topo do moirão.

\subsection{Amostragem dos moirões tratados}

Para assegurar uma boa fixação dos sais preservativos na madeira, os moirões tratados foram submetidos à secagem ao ar, durante 30 dias, em local coberto e ventilado. Após a secagem, retiraram-se discos de 2,0 cm de espessura em quatro posições ao longo dos moirões (Figura 1). Com os discos, foram realizadas as análises de penetração, distribuição e retenção do CCB.

A análise de retenção foi realizada apenas com o disco proveniente da posição 1 , uma vez que esta coincide com a região de afloramento em moirões instalados no solo. Nas demais posições, foram avaliadas a penetração e a distribuição dos elementos cobre e boro. Desta forma, procurou-se representar a penetração e a distribuição do preservativo no sentido medula-casca e na direção longitudinal das peças tratadas.

Para as análises de retenção do preservativo, de cada disco, foram obtidas amostras de $1,0 \times 1,0 \times 2,5 \mathrm{~cm}$, em sentidos opostos (Figura 2). Em função do custo das análises de retenção e da necessidade de representatividade, foram empregados dois moirões que tinham características semelhantes, para cada tratamento.

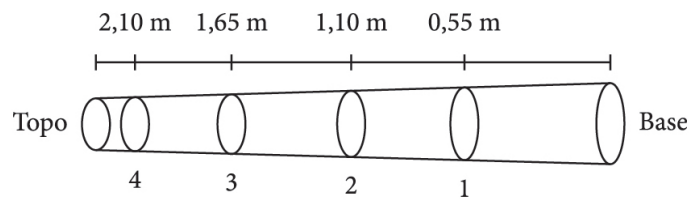

Figura 1. Posições nos moirões das quais foram retirados os discos para as análises de penetração, distribuição e retenção de CCB.

Figure 1. Positions in fence posts where the disks were taken for the analyses of penetration, distribution and retention of CCB.

Tabela 1. Quantidade de produto químico utilizado de acordo com a concentração empregada, para 100 litros de água.

Table 1. Quantity of used chemistry product in according to the concentration employed, for 100 liters of water.

\begin{tabular}{lcccc}
\multirow{2}{*}{ Produto Químico } & \multicolumn{4}{c|}{ Massa (g) de Produto Químico por Concentração (\%) } \\
\cline { 2 - 5 } & $\mathbf{0 , 5}$ & $\mathbf{1 , 0}$ & $\mathbf{2 , 0}$ & $\mathbf{3 , 0}$ \\
\hline Dicromato de potássio & 400,23 & 800,41 & $1.600,80$ & $2.401,20$ \\
\hline Ácido bórico & 252,03 & 504,07 & $1.008,14$ & $1.512,18$ \\
\hline Sulfato de cobre & 347,75 & 695,51 & $1.351,00$ & $2.081,55$ \\
\hline
\end{tabular}




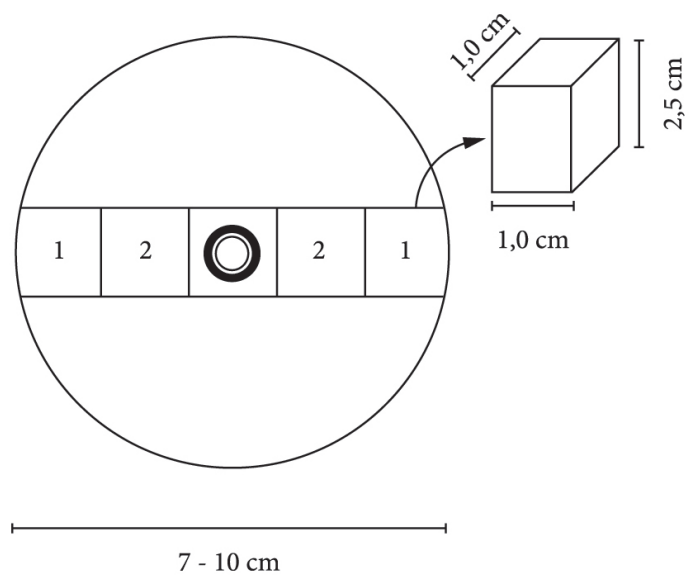

Figura 2. Posições nos discos das quais foram retiradas as amostras para análises de retenção do CCB.

Figure 2. Positions on the disks where the samples were taken for analysis of CCB retention.

\subsection{Análises químicas da madeira tratada}

Para determinar a penetração dos elementos cobre e boro na madeira tratada, foi realizada análise colorimétrica, seguindo as recomendações da NBR 6232 (ABNT, 2013). Para a determinação do elemento cobre, os discos foram pincelados com solução de cromo-azurol S, que revela uma coloração azul escuro intensa na presença desse elemento. Para o boro, os discos foram pincelados com uma solução de álcool polivinílico e iodo, revelando uma coloração azulada na presença do elemento.

Para estas determinações, demarcaram-se, aleatoriamente, duas linhas perpendiculares, passando pela medula, sobre as quais foram medidas as profundidades de penetração em milímetros, em quatro seções no disco. O valor médio das medições foi utilizado para avaliar a penetração dos elementos cobre e boro, em cada posição nos moirões. A distribuição foi avaliada com base nas médias das medidas de penetração ao longo do comprimento de cada moirão.

Para a determinação da retenção do CCB, efetuou-se a digestão das amostras obtidas (Figura 2), seguindo a metodologia descrita por Farias Sobrinho et al. (2005), Paes et al. $(2005,2012)$ e Torres et al. (2011). A metodologia consta da determinação do volume das amostras e incineração, para obtenção das cinzas e sais metálicos, a $500-550^{\circ} \mathrm{C}$; posteriormente, foram adicionados
$3 \mathrm{~mL}$ da mistura dos ácidos sulfúrico, perclórico e nítrico, todos concentrados, nas proporções de 7:2:1, às cinzas obtidas pela incineração. Em seguida, foi realizada a digestão acelerada pelo aquecimento da mistura dos ácidos e cinzas, em chapas aquecidas, até a mistura ficar límpida, e realizada a diluição das soluções ácidas em água destilada a volumes fixos de $100 \mathrm{~mL}$ e armazenadas em frascos plásticos devidamente identificados.

A leitura do elemento cobre foi no Laboratório de Análises de Fertilizantes, Águas, Minérios, Resíduos, Solos e Plantas da Universidade Federal do Espírito Santo e a dos elementos cromo e boro, no Laboratório de Espectrometria Atômica, do Departamento de Solos da Universidade Federal de Viçosa. A leitura dos elementos cobre e cromo deu-se por espectrometria de absorção atômica e a do boro, por fotometria de chama. Com os dados obtidos, foram efetuados os cálculos da retenção, empregando a Equação 1, citada por Paes et al. $(2005,2012)$ e Torres et al. (2011).

$R=\frac{F \times L \times F d \times 10^{-3}}{V}$

Em que:

$\mathrm{R}=$ Retenção do elemento na madeira (kg de i.a.m ${ }^{-3}$ );

$\mathrm{F}=$ Fator estequiométrico empregado para transformação dos elementos químicos para óxidos $\left(\mathrm{Cu} \times 1,2518=\mathrm{CuO} ; \mathrm{Cr} \times 1,9230=\mathrm{CrO}_{3}\right) ;$

$\mathrm{L}=$ Leitura obtida do espectrofotômetro ou fotômetro de chama (mg. $\left.\mathrm{L}^{-1}\right)$;

Fd = Fator de diluição necessário para as leituras dos elementos químicos;

$\mathrm{V}=$ Volume das amostras utilizadas nas análises $\left(\mathrm{cm}^{3}\right)$.

\subsection{Análise estatística dos resultados}

Com referência à determinação do comprimento e dos diâmetros médios dos moirões, realizaram-se cálculos estatísticos simples, em que os valores da média e do desvio padrão foram determinados.

Para o tratamento das peças, uma vez que houve homogeneidade dos dados e os mesmos seguiram uma distribuição normal, foi utilizado um delineamento inteiramente casualizado no 
esquema fatorial, contendo quatro concentrações $(0,5 ; 1,0 ; 2,0 ; 3,0 \%)$. A penetração foi avaliada para os elementos cobre e boro em quatro posições nos moirões (Figura 1). Para a análise de retenção, foram avaliados os fatores posição no disco (Figura 2) e a concentração da solução de tratamento. Na avaliação do experimento, foi empregado o teste de Tukey, em nível de $5 \%$ de significância, para as fontes de variação detectadas como significativas pelo teste de F.

\section{RESULTADOS E DISCUSSÃO}

Como observado na Tabela 2, as temperaturas mais elevadas foram observadas às 16 horas (média de $28^{\circ} \mathrm{C}$ ) e as mais amenas, às 20 horas (média de $21^{\circ} \mathrm{C}$ ). O tratamento dos moirões pode ter sido mais efetivo no período compreendido entre $12 \mathrm{e}$ 16 horas, uma vez que foram observados os menores valores de umidade relativa, sendo menos efetivo no período da noite $(20 \mathrm{~h})$ e, no período da manha ( $8 \mathrm{~h}$ ), quando foram observados os maiores valores de umidade relativa.

Os moirões submetidos ao tratamento com 0,5\% de i.a. ficaram expostos a essas condições até o oitavo dia de tratamento; os tratados com 1,0\% até o sétimo dia; aqueles tratados com $2,0 \%$ ficaram expostos a essas condições por cinco dias, e os tratados com $3,0 \%$, quatro dias. Os tratamentos eram finalizados à medida que a solução preservativa atingia o topo dos moirões.

A influência da concentração no tempo de tratamento da madeira é um parâmetro importante para as usinas de tratamento, estabelecendo referências no controle da qualidade de moirões tratados.
A NBR 9480, ABNT (2009) recomenda que a penetração do produto preservativo deva ser total no alburno (madeiras de folhosas); porém, em se tratando de tratamento não industrial, dificilmente se obtém a penetração total do alburno ao longo das peças. Dessa forma, logo se adotou uma penetração mínima de $10 \mathrm{~mm}$ (Paes et al., 2005, Torres et al., 2011).

Os moirões tinham dimensões semelhantes, com variações em seu comprimento de 2,19 a 2,23 m, e o diâmetro de 7,02 a 9,43 cm, com média de $8,05 \mathrm{~cm}$ (Tabela 3). Há a necessidade de se homogeneizar ao máximo as características dos moirões para que as mesmas não venham a influenciar na ascensão da solução preservativa nos mesmos.

Verifica-se, na Figura 3, que ocorreu a diminuição dos dias de tratamento da madeira, na medida em que aumentou a concentração de ingredientes

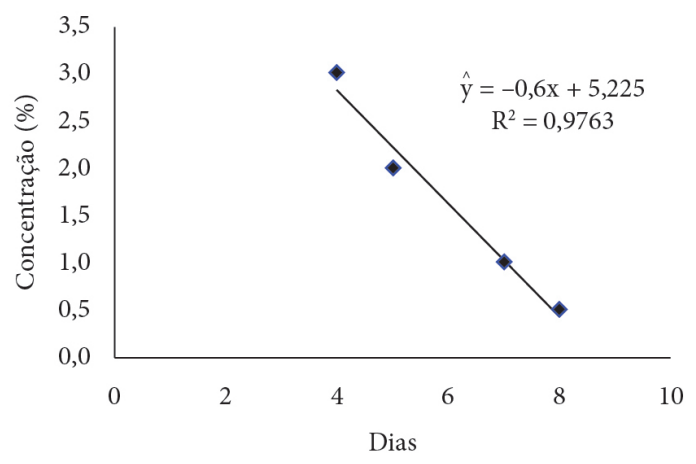

Figura 3. Tempo de ascensão da solução preservativa nos moirões.

Figure 3. Ascension time of the preservative solution in fence posts.

Tabela 2. Condições climatológicas locais durante o tratamento da madeira.

Table 2. Locals weather condition during the wood treatment.

\begin{tabular}{|c|c|c|c|c|c|c|c|c|}
\hline \multirow{3}{*}{$\begin{array}{c}\text { Dia do } \\
\text { Tratamento }\end{array}$} & \multicolumn{8}{|c|}{ Horário de Tomada das Leituras } \\
\hline & \multicolumn{4}{|c|}{ Temperatura $\left({ }^{\circ} \mathrm{C}\right)$} & \multicolumn{4}{|c|}{ Umidade Relativa (\%) } \\
\hline & 8 & 12 & 16 & 20 & 8 & 12 & 16 & 20 \\
\hline 1 & - & - & 24 & 21 & - & - & 55 & 83 \\
\hline 2 & 17 & 23 & 25 & 22 & 90 & 84 & 51 & 84 \\
\hline 3 & 18 & 25 & 29 & 21 & 90 & 64 & 38 & 75 \\
\hline 4 & 23 & 28 & 29 & 21 & 62 & 48 & 43 & 67 \\
\hline 5 & 22 & 27 & 27 & 20 & 61 & 53 & 59 & 58 \\
\hline 6 & 24 & 28 & 28 & 20 & 63 & 42 & 48 & 58 \\
\hline 7 & 23 & 27 & 29 & 21 & 62 & 59 & 43 & 60 \\
\hline 8 & 24 & - & - & - & 55 & - & - & - \\
\hline Média & 23 & 27 & 28 & 21 & 69 & 58 & 48 & 69 \\
\hline
\end{tabular}


ativos. Isto pode ser explicado, pois as soluções mais concentradas se difundem mais rapidamente no lenho do que as soluções menos concentradas (processo osmótico) (Paes et al., 2005).

A distribuição do boro nos moirões está apresentada na Figura 4. Verificou-se que o boro se distribuiu de forma mais homogênea do que o cobre (Figura 5) em todas as peças submetidas ao tratamento. Os resultados da penetração do boro estão dispostos na Tabela 4.

A maior penetração observada foi de $12,41 \mathrm{~mm}$ no moirão 16 , de concentração $3,0 \%$, seguida pelo moirão 14, de mesma concentração. A menor penetração foi de $6,77 \mathrm{~mm}$ no moirão 17 , de concentração $0,5 \%$.

Para as concentrações de 0,5 e 1,0\%, o tempo de tratamento não foi suficiente para assegurar a utilização destes moirões em contato com o solo, uma vez que a penetração do elemento boro foi satisfatória apenas para três moirões (moirão 12, 2,0\% de concentração, e nos moirões, 14 e 16, 3,0\% de solução preservativa) (Tabela 3 ). As menores penetrações foram atingidas nos moirões em que a concentração utilizada foi de $0,5 \%$, tendo valores médios de 7,65 e 6,77 mm, nos moirões 4 e 17, respectivamente.

Tabela 3. Diâmetro e comprimento dos moirões para cada posição e concentração da solução de tratamento. Table 3. Fence posts diameter and length for each position and treatment solution concentration.

\begin{tabular}{|c|c|c|c|c|c|c|c|}
\hline \multirow{2}{*}{$\begin{array}{c}\text { Concentração } \\
(\%)\end{array}$} & \multirow{2}{*}{ Moirão } & \multirow{2}{*}{$\begin{array}{c}\text { Comprimento } \\
(\mathrm{m})\end{array}$} & \multicolumn{4}{|c|}{ Diâmetro dos Discos em cada Posição $(\mathrm{cm})$} & \multirow{2}{*}{$\begin{array}{c}\text { Média } \\
(\mathrm{cm})\end{array}$} \\
\hline & & & 1 & 2 & 3 & 4 & \\
\hline \multirow{2}{*}{0,5} & 4 & 2,19 & 10,12 & 9,32 & 9,19 & 9,08 & 9,43 \\
\hline & 17 & 2,20 & 7,69 & 7,27 & 7,17 & 6,88 & 7,24 \\
\hline \multirow{2}{*}{1,0} & 13 & 2,19 & 8,22 & 8,21 & 7,83 & 7,55 & 7,95 \\
\hline & 5 & 2,19 & 8,87 & 8,50 & 8,49 & 8,06 & 8,48 \\
\hline \multirow{2}{*}{2,0} & 12 & 2,20 & 9,30 & 9,09 & 8,70 & 8,30 & 8,85 \\
\hline & 6 & 2,23 & 7,53 & 7,36 & 7,29 & 7,03 & 7,30 \\
\hline \multirow{2}{*}{3,0} & 16 & 2,20 & 7,88 & 8,07 & 8,13 & 8,30 & 8,09 \\
\hline & 14 & 2,20 & 7,28 & 7,01 & 6,96 & 6,80 & 7,02 \\
\hline \multicolumn{2}{|c|}{ Média } & 2,20 & 8,48 & 8,14 & 7,99 & 7,81 & 8,05 \\
\hline \multicolumn{2}{|c|}{ Desvio Padrão } & 0,01 & 0,98 & 0,85 & 0,80 & 0,82 & 0,85 \\
\hline
\end{tabular}

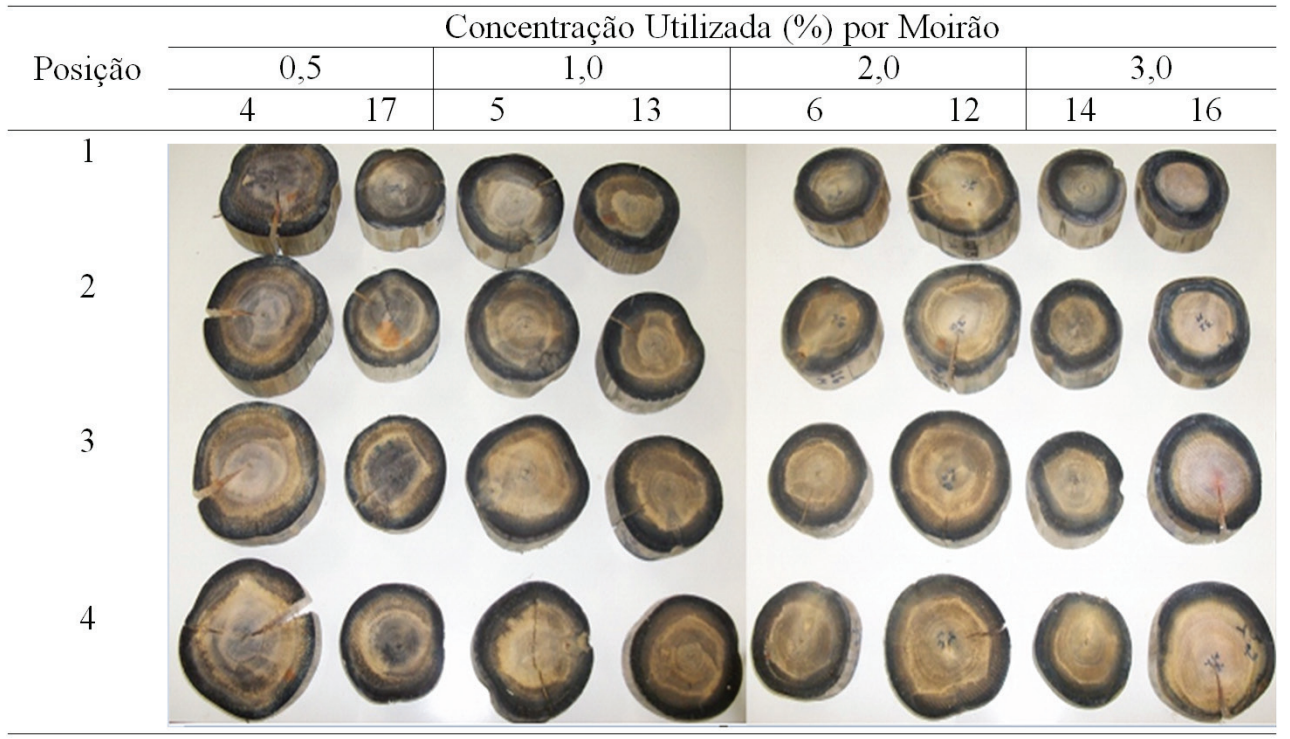

Figura 4. Distribuição do boro nos moirões de acordo com a posição e a concentração. Figure 4. Boron distribution in fence posts according to position and concentration. 


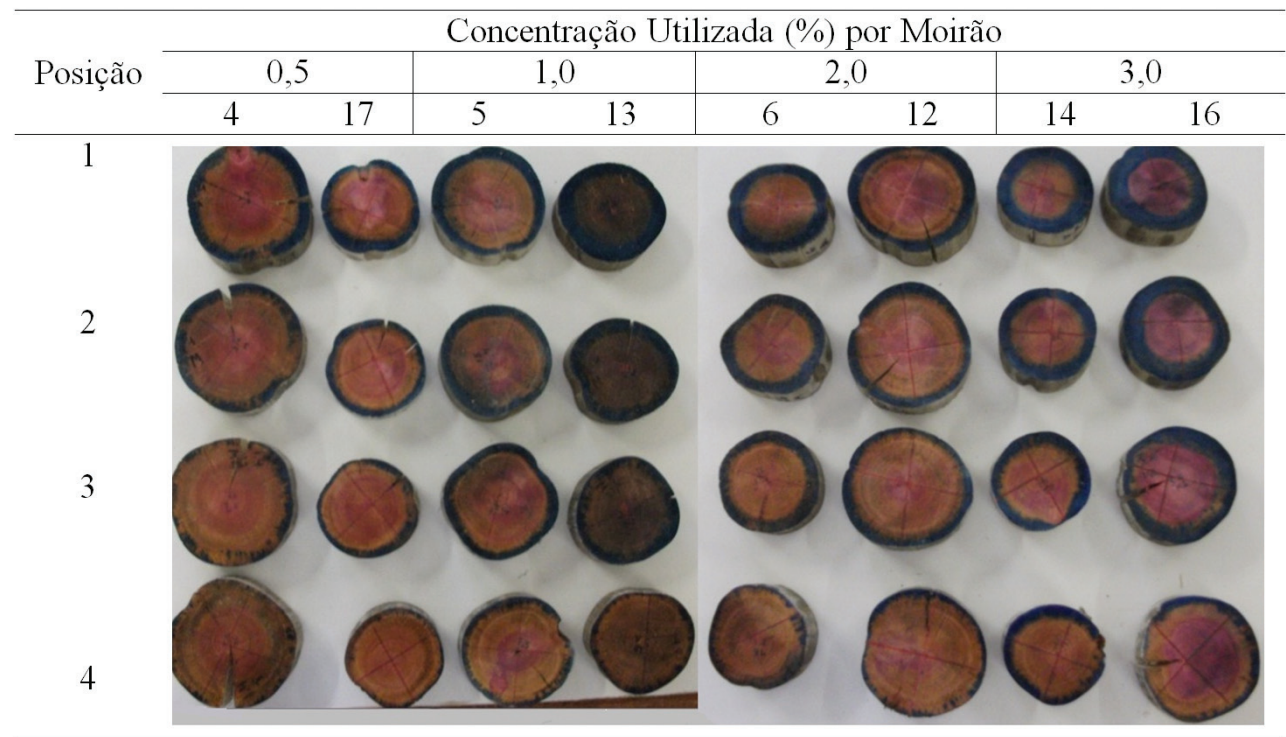

Figura 5. Distribuição do cobre nos moirões de acordo com a posição e a concentração.

Figure 5. Cupper distribution in fence posts according to position and concentration.

Tabela 4. Penetração de boro para as concentrações, os tempos de tratamento e as posições nos moirões.

Table 4. Boron penetration to concentrations, treatment times and positions in fence posts.

\begin{tabular}{|c|c|c|c|c|c|c|c|}
\hline \multirow{2}{*}{$\begin{array}{c}\text { Concentração } \\
(\%)\end{array}$} & \multirow{2}{*}{$\begin{array}{l}\text { Tempo } \\
\text { (Dias) }\end{array}$} & \multirow{2}{*}{$\begin{array}{c}\text { Moirão } \\
\text { (Número) }\end{array}$} & \multicolumn{4}{|c|}{ Penetração de Boro (mm) por Posição nos Moirões } & \multirow{2}{*}{$\begin{array}{c}\text { Médias } \\
(\mathrm{mm})\end{array}$} \\
\hline & & & 1 & 2 & 3 & 4 & \\
\hline \multirow{2}{*}{0,5} & \multirow{2}{*}{8} & 4 & 8,84 & 7,98 & 7,31 & 6,68 & 7,65 \\
\hline & & 17 & 9,40 & 7,48 & 6,06 & 5,62 & 6,77 \\
\hline \multirow{2}{*}{1,0} & \multirow{2}{*}{6} & 13 & 9,91 & 8,31 & 8,82 & 10,12 & 9,36 \\
\hline & & 5 & 9,24 & 8,28 & 9,79 & 10,19 & 9,52 \\
\hline \multirow{2}{*}{2,0} & \multirow{2}{*}{5} & 6 & 9,95 & 9,16 & 10,21 & 9,18 & 9,57 \\
\hline & & 12 & 9,92 & 9,80 & 11,71 & 11,88 & 10,81 \\
\hline \multirow{2}{*}{3,0} & \multirow{2}{*}{4} & 16 & 14,40 & 11,82 & 11,45 & 9,87 & 11,63 \\
\hline & & 14 & 15,85 & 13,95 & 10,88 & 9,56 & 12,41 \\
\hline
\end{tabular}

A análise estatística aplicada aos dados indicou que houve diferença significativa para as posições nas peças, as concentrações das soluções e a interação entre posição e concentração a 1\%, pelo teste de F. Foi elaborado o desdobramento da interação e realizada a comparação das médias pelo Teste de Tukey a 5\% de significância (Tabela 5).

Conforme observado na Tabela 5, as posições 1 (zona de afloramento) e 2 tiveram as melhores médias, tendo estas não diferido entre si, pelo teste de Tukey a $5 \%$ de significância. Também se pode verificar que a concentração de $\mathrm{CCB}$ a 3,0\% teve as melhores médias para todas as posições; observou-se que, quando comparada às concentrações entre as posições, a posição 4 obteve as piores penetrações.
A existência de interação para o boro, não observada para o cobre, deve-se, provavelmente, ao maior poder de difusão das moléculas de boro (Paes et al., 2005).

Nota-se que o cobre penetrou em todos os discos (Figura 5), mas de forma diferente em cada tratamento e posição, tendo a penetração decrescido da base para o topo, não atingindo, nesta posição, em nenhuma das peças, a penetração de $10 \mathrm{~mm}$, considerada por Paes et al. $(2005,2012)$ e Torres et al. (2011) como a penetração mínima necessária para proteger a madeira. Observa-se, na Tabela 6, que a penetração foi maior na posição 1 (zona de afloramento), com redução ao longo do moirão, para todas as concentrações testadas. A maior penetração foi de $11,68 \mathrm{~mm}$, no moirão 16 (3,0\% concentração 
Tabela 5. Comparações entre as médias de penetração de boro para as concentrações e posições nos moirões tratados.

Table 5. Comparisons among averages of boron penetration for concentrations and positions in treated fence posts.

\begin{tabular}{ccccc} 
Concentração & \multicolumn{4}{c}{ Penetração Média $(\mathbf{m m})$ por Posição nos Moirões } \\
\cline { 2 - 5 }$(\%)$ & $\mathbf{1}$ & $\mathbf{2}$ & $\mathbf{3}$ & $\mathbf{4}$ \\
\hline 0,5 & $9,12 \mathrm{Ab}$ & $7,73 \mathrm{ABb}$ & $6,62 \mathrm{Bb}$ & $6,15 \mathrm{Bb}$ \\
1,0 & $9,58 \mathrm{Ab}$ & $8,30 \mathrm{Ab}$ & $9,31 \mathrm{Aa}$ & $10,16 \mathrm{Aa}$ \\
2,0 & $9,93 \mathrm{Ab}$ & $9,48 \mathrm{Ab}$ & $10,96 \mathrm{Aa}$ & $10,53 \mathrm{Aa}$ \\
3,0 & $15,13 \mathrm{Aa}$ & $12,89 \mathrm{ABa}$ & $11,17 \mathrm{BCa}$ & $9,74 \mathrm{Ca}$ \\
\hline
\end{tabular}

Médias seguidas pela mesma letra maiúscula na horizontal ou minúscula na vertical não diferem entre si (Tukey; $\mathrm{p} \geq 0,05$ ).

Tabela 6. Penetração de cobre para as concentrações, os tempos de tratamento e as posições nos moirões.

Table 6. Cupper penetration to concentrations, treatment times and positions in fence posts.

\begin{tabular}{|c|c|c|c|c|c|c|c|}
\hline \multirow{2}{*}{$\begin{array}{c}\text { Concentração } \\
(\%)\end{array}$} & \multirow{2}{*}{$\begin{array}{l}\text { Tempo } \\
\text { (Dias) }\end{array}$} & \multirow{2}{*}{$\begin{array}{c}\text { Moirão } \\
\text { (Número) }\end{array}$} & \multicolumn{4}{|c|}{ Penetração de Cobre (mm) por Posição nos Moirões } & \multirow{2}{*}{$\begin{array}{l}\text { Média } \\
(\mathrm{mm})\end{array}$} \\
\hline & & & 1 & 2 & 3 & 4 & \\
\hline \multirow{2}{*}{0,5} & \multirow{2}{*}{8} & 4 & 7,77 & 7,01 & 3,39 & 2,57 & 5,18 \\
\hline & & 17 & 7,22 & 6,16 & 5,08 & 1,95 & 5,10 \\
\hline \multirow{2}{*}{1,0} & \multirow{2}{*}{6} & 13 & 8,57 & 8,14 & 6,79 & 3,84 & 7,04 \\
\hline & & 5 & 8,73 & 8,01 & 7,95 & 3,47 & 6,83 \\
\hline \multirow{2}{*}{2,0} & \multirow{2}{*}{5} & 6 & 9,34 & 8,26 & 7,96 & 4,34 & 7,47 \\
\hline & & 12 & 9,47 & 8,29 & 7,85 & 3,71 & 7,33 \\
\hline \multirow{2}{*}{3,0} & \multirow{2}{*}{4} & 14 & 12,92 & 9,19 & 6,86 & 6,66 & 8,91 \\
\hline & & 16 & 14,95 & 14,94 & 12,43 & 5,17 & 11,68 \\
\hline
\end{tabular}

de i.a.), e a menor penetração média foi de $5,10 \mathrm{~mm}$ no moirão 4 , na solução de $0,5 \%$.

Os resultados corroboram com a necessidade de maior tempo de tratamento dos moirões nas soluções preservativas de $0,5,1,0$ e $2,0 \%$, e a sua posterior inversão, para homogeneizar a penetração de cobre ao longo do moirão, como citado por Torres et al. (2011).

A maior penetração ocorreu na posição 1 (zona de afloramento), fato que pode ter ocorrido em virtude da parte inferior ter permanecido submersa na solução preservativa, enquanto a superior se manteve fora da solução. Este comportamento foi observado por Torres et al. (2011), para moirões de Eucalyptus camaldulensis, e por Paes et al. (2005), para Eucalyptus viminalis, submetidos ao processo de substituição de seiva por transpiração radial. Observa-se um incremento da penetração deste elemento, na medida em que houve o aumento da concentração da solução.

O tempo de ascensão, na solução de 3,0\%, foi suficiente para suprir a demanda de cobre (fungicida) na zona de afloramento (posição 1), recomendando-se seu uso em contado direto com o solo, pois este elemento possui eficiência contra fungos apodrecedores, condições estas que expõem as madeiras ao ataque destes organismos.

$\mathrm{O}$ teste de $\mathrm{F}$ indicou que existe diferença significativa entre as variáveis posição e concentração. Assim, o teste de médias de Tukey foi aplicado a tais fatores a 5\% de significância. Os resultados do teste de média aplicado e suas comparações encontram-se na Tabela 7 .

A análise da média indicou que as posições 1 e 2 foram superiores às demais, e na posição 4, foram obtidas as menores médias, o que pode ser explicado pela baixa umidade no topo dos moirões e pela posição 1 estar imersa na solução, enquanto as demais encontrarem-se fora deste contato (Farias Sobrinho et al., 2005; Paes et al., 2005).

Notou-se que as maiores penetrações foram obtidas em concentrações de 3,0\%, e as menores, obtidas na de $0,5 \%$. Conforme citado por Brazolin (2007), as características do processo de tratamento e da espécie de madeira utilizada influenciam na qualidade do tratamento.

Observou-se que a posição influenciou na retenção do preservativo, tendo as amostras externas 
Tabela 7. Comparações entre as médias de penetração de cobre para as posições e concentrações nos moirões tratados.

Table 7. Comparisons among averages of cupper penetration for positions and concentrations in treated fence posts.

\section{Posição}

1

2

3

4
Penetração $(\mathrm{mm})$

$10,00 \mathrm{a}$

$8,55 \mathrm{ab}$

$7,45 \mathrm{~b}$

$3,96 \mathrm{c}$

\section{Concentração (\%)}

0,5

1,0

2,0

3,0
Penetraç̃o $(\mathrm{mm})$

$10,30 \mathrm{a}$

$7,40 \mathrm{~b}$

$7,06 \mathrm{bc}$

$5,14 \mathrm{c}$

Médias seguidas pela mesma letra, em cada parâmetro, não diferem estatisticamente pelo teste de Tukey ( $\mathrm{p} \geq 0,05)$.

maiores retenções, e as internas, menores retenções (Figuras 6 e 7, respectivamente).

Na Figura 6, nota-se que o tempo de tratamento de 8 dias (Figura 3) para a solução preservativa de $0,5 \%$ não foi suficiente para atender ao mínimo recomendado pela NBR 9480, ABNT (2009), que é de $6,5 \mathrm{~kg}$ de i.a.m $\mathrm{m}^{-3}$. De acordo com Farias Sobrinho et al. (2005), madeiras com retenção de 4,0 a 6,5 kg de i.a.m ${ }^{-3}$ podem ser utilizadas em estruturas fora do contato direto com o solo, desde que seja aplicado um reforço no topo das mesmas; contudo, este fato não foi atendido pelos moirões nesta concentração utilizada.

Notou-se que os dias de tratamento para os demais moirões (Figura 3) foram suficientes para que se atingisse a retenção mínima recomendada pela normatização (Figura 6). Com isto, as peças podem ser empregadas em contato direto com o solo.

$\mathrm{Na}$ Figura 7, pode-se observar que, em nenhuma das concentrações, as amostras internas atingiram o mínimo recomendado pela NBR 9480, ABNT (2009). Isto pode ser explicado pela dificuldade na difusividade do produto preservativo e pelo teor de cerne e alburno nas peças. Pode ser observado que os valores de retenção cresceram na medida em que houve o aumento da concentração dos ingredientes ativos e do tempo de tratamento. Desta forma, para se obterem melhores retenções nas camadas mais internas das peças, o tempo de tratamento terá de ser maior do que o proposto neste trabalho.

A análise estatística aplicada aos dados indicou que não houve diferença significativa a $5 \%$ de significância pelo teste de $\mathrm{F}$ entre a posição da amostra retirada no disco e a concentração utilizada. Isto pode ser explicado pelo baixo valor do resíduo obtido na análise estatística (Nesi et al., 2010).

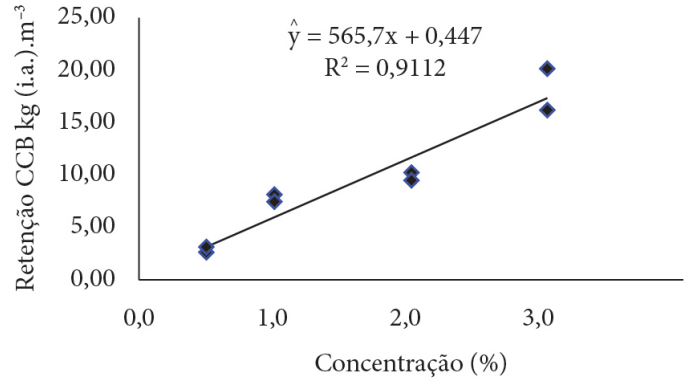

Figura 6. Efeito da concentração da solução na retenção de CCB para as amostras externas.

Figure 6. Effect of solution concentration in $\mathrm{CCB}$ retention to external samples.

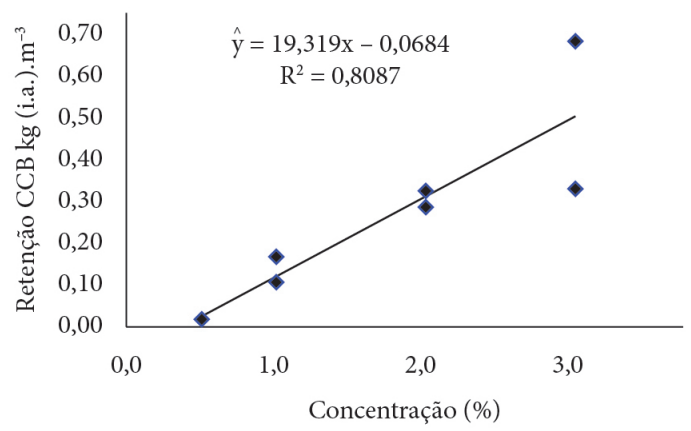

Figura 7. Efeito da concentração da solução na retenção de CCB para as amostras internas.

Figure 7. Effect of solution concentration in CCB retention to internal samples.

\section{CONCLUSÕES}

O tempo de ascensão dos produtos preservativos influenciou na qualidade do tratamento de madeiras, nos aspectos de distribuição, penetração e retenção.

Os índices de penetração de cobre, para o tempo de tratamento na ascensão do produto preservativo, foram satisfatórios apenas para os moirões tratados 
com 3,0\% de solução preservativa, atingindo valores próximos em soluções de 2,0\%.

A análise de retenção do produto preservativo mostrou que apenas os moirões tratados com $0,5 \%$ de concentração não atenderam às recomendações mínimas estabelecidas pela normatização de peças tratadas com produtos hidrossolúveis.

O tempo de tratamento dos moirões não foi suficiente para que as amostras internas na análise de retenção atendessem à legislação brasileira.

O tempo de tratamento para as soluções de 0,5 ; 1,0 e 2,0\% de i.a., respectivamente, 8, 7 e 5 dias, não foi suficiente para que os moirões atingissem a recomendação da legislação brasileira. Portanto, recomenda-se maior tempo de tratamento para estas soluções.

\section{AGRADECIMENTOS}

Ao Professor Onofre Dardengo, proprietário da propriedade rural Providência, por ter cedido a madeira empregada no estudo.

\section{STATUS DA SUBMISSÃO}

Recebido: 30 mar., 2013

Aceito: 19 ago., 2013

Publicado: 28 ago., 2014

\section{AUTOR(ES) PARA CORRESPONDÊNCIA}

Juarez Benigno Paes

Departamento de Ciências Florestais e da Madeira, Universidade Federal do Espírito Santo - UFES, CEP 29550-000, Jerônimo Monteiro, ES, Brasil e-mail: jbp2@uol.com.br

\section{REFERÊNCIAS}

Associação Brasileira de Normas Técnicas - ABNT. NBR 6232: penetração e retenção de preservativos em madeira tratada sob pressão. Rio de Janeiro; 2013.

Associação Brasileira de Normas Técnicas - ABNT. NBR 9480: peças roliças preservadas de eucalipto para construções rurais. Requisitos. Rio de Janeiro; 2009.

Brazolin S. Biodeterioração e preservação de madeira. In: Oliveira JTS, Fiedler NC, Nogueira M, editores. Tecnologias aplicadas ao setor madeireiro. Visconde do Rio Branco: Suprema Gráfica e Editora; 2007.

Farias Sobrinho DW, Paes JB, Furtado DA. Tratamento preservativo da madeira de algaroba (Prosopolis julifora (Sw) D. C.), pelo método de substituição de seiva. Cerne 2005; 11(3): 225-236.

Lelles JG, Rezende JLP. Considerações gerais sobre tratamento preservativo da madeira de eucalipto. Informe Agropecuário 1986; 12(141): 83-90.

Magalhães WLE, Pereira JCD. Método de substituição de seiva para preservação de mourões. Colombo: EMBRAPA; 2003. Comunicado Técnico n. 97.

Nesi CN, Guidoni AL, Bringhenti C. O problema do baixo número de repetições em experimentos de competição de cultivares. Revista de Ciências Agroveterinárias 2010; 9(1): 74-81.

Paes JB, Moreschi JC, Lelles JG. Avaliação do tratamento preservativo de moirões de Eucalyptus viminalis Lab. e de bracatinga (Mimosa scarabella Benth.) pelo método de substituição de seiva. Ciência Florestal 2005; 15(1): 75-86.

Paes JB, Lombardi LR, Oliveira, JTS, Silva, LF, Santos, LL. Qualidade de moirões de eucalipto tratados comercializados em três municípios do Espírito Santo. Floresta e Ambiente 2012; 19(4): 475-482. http://dx.doi. org/10.4322/floram.2012.048

Paes JB, Vital BR. Resistência natural da madeira de cinco espécies de eucalipto a cupins subterrâneos em teste de laboratório. Revista Árvore 2000; 24(1): 1-6.

Torres PMA, Paes JB, Lira Filho JA, Nascimento JWB. Tratamento preservativo da madeira juvenil de Eucalyptus camaldulensis Dehuh. pelo método de substituição de seiva. Cerne 2011; 17(2): 275-282. 